Original Research Paper

\title{
Explosion of the Light: Alliance of the Planck and Mach and Einstein-Riemannian Physics
}

\author{
Mohsen Lutephy \\ Department of Chemistry, Islamic Azad University (IAU), Tehran, Iran
}

\section{Article history}

Received: $15-08-2019$

Revised: 28-11-2019

Accepted: 03-12-2019

Email: lutephy@gmail.com

\begin{abstract}
On the evident tripartite equivalency of the light speed and time and distance as a natural paradigm introduced here it is deduced the natural metric tensor of the universe via Mach's mechanics (metric fully governed by measurable universe) included to the fully variable light speed and fully variable gravitational $\mathrm{G}$ accompanied with Planckian quantum gravity in natural quantization of the space-time and we see the quantum modes of the gravitational fields by combination of the Planck and Mach and EinsteinRiemannian physics introducing new universal constants. We see a new cosmology on the base of the variable light speed universe via conservation of the total matter of the universe expanding stationary via permanent cyclical transformation of the observable baryonic matter to background ether and the background ether to the observable baryonic matter required by equation of the continuity for equilibrium, creating homogeneity and isotropy and flattening of the universe and reasoning also a universal background minimum temperature creating cosmic microwave background as a black body radiation. Ultimately it is obvious that our universe is a large version of the Planckian mini universe and also we see variable Planck length correlated to the gravitational potential energy.
\end{abstract}

Keywords: Quantum Gravity, Cosmology, Planck Units, Planck Stars, Machian Universe, Variable Light Speed, Quantization of the Space-Time

\section{Introduction}

Quantum Gravity, broadly construed, is a physical theory incorporating both the gravity and quantum theory. Such a theory is expected to be able to provide a satisfactory description of the microstructure of spacetime at the so-called Planck scale, at which all fundamental constants of the ingredient theories, $\mathrm{c}$ (the velocity of light), $\hbar$ (the reduced Planck's constant) and $\mathrm{G}$ (Newton's constant), come together to form units of mass, length and time. The Planck length and time units are so small capable to be measured experimentally.

The Planck (1899) demonstrated a natural absolute family of units that are independent of all human and terrestrial baggage. Planck noted that in all ordinary systems of units, the choice of the basic units is made not from a general point of view "necessary for all places and times," but is determined solely by "the special needs of our terrestrial culture" (Planck, 1899, p. 479).

Planck was not aware of the relevance of the scale set by the constants to the applicability of general relativity, of course, but Arthur Eddington seems to have been aware, writing in the March edition of Nature in 1918:

\begin{abstract}
"From the combination of the fundamental constants, $G, c$ and $h$ it is possible to form a new fundamental unit of length $L_{\min }=7 \times 10^{-28}$ $\mathrm{cm}$. It seems to be inevitable that this length must play some role in any complete interpretation of gravitation. ... (Eddington, 1918, p. 36)”.
\end{abstract}

The idea of a minimal length was predated by a smallest unit of time, proposed by Lévi (1927) in his "Hyphoth 'ese de l'atome de temps" (hypothesis of time atoms), that was further developed by Pokrowski (1928).

It was not until special relativity and quantum mechanics were joined in the framework of quantum field theory that the possible existence of a minimal length scale rose to the awareness of the community.

The idea that the Planck length amounts to a minimal length in nature follows from the argument that if distances smaller than this length are resolved, then it would require energies concentrated in a region so small that a mini-black hole would form, taking the observed system with it (Rovelli, 2007, pp. 1289). 
Heisenberg was among the first to consider a fundamentally discrete space-time that would yield a cutoff, laid out in his letters to Bohr and Pauli. But young Russian, Bronstein (1936) was first to comprehend the problem of quantizing gravity in the Planck scale.

It was not until 1964, that Mead $(1964 ; 1966)$ pointed out the peculiar role that gravity plays in our attempts to test physics at short distances. He showed, in a series of thought experiments that this influence does have the effect of amplifying Heisenberg's measurement uncertainty, making it impossible to measure distances to a precision better than Planck's length. Up to date 1960s, his argument for the fundamental relevance of the Planck length met strong resistance.

But in the mid-1970s then Hawking's calculation (1975) of a black hole's thermodynamical properties introduced the 'transplanckian problem' due to the, in principle infinite, blue shift of photons approaching a black hole horizon, modes with energies exceeding the Planck scale had to be taken into account to calculate the emission rate. However, the prominent role played by John Wheeler, whose contributions, though not directly on the topic of a minimal length, has connected blackhole physics with space-time foam and the Planckian limit and by this inspired much of what followed.

Unruh (1995) suggested that one use a modified dispersion relation to deal with the difficulty of transplanckian modes, so that a smallest possible wavelength takes care of the contributions beyond the Planck scale. A similar problem exists in inflationary cosmology (Martin, 2001) since tracing back in time small frequencies increases the frequency till it eventually might surpass the Planck scale at which point we no longer know how to make sense of general relativity. the idea of a fundamentally finite length or a maximum frequency was in these years studied by many scientists but here we have shown a meaningful connection between gravitation and fundamental minimum length.

Einstein relativity was not ultimately achieved with Mach inertia principle (metric fully governed by universe) because of non-relativistic attachments into the general relativity and we see here that the time is not an independent parameter in the physics suppose the time is light speed equivalent as the meter is.

Einstein total field is arbitrary selection without a natural argument whereas we want to find a natural metric not in paradox, fully compatible with nature evidences. Arbitrary choice of an equation for physics is not forbidden mathematically but sometimes the mathematical concepts are in conflict with evidences of the physical nature so that the differential equations of the mathematics results the famous Zeno's paradox and even in the mathematics too, the continuity of the real numbers is impossible however ever for two numbers we can consider a number between them in an infinity consequent process which is violating continuum motion.

Julian Barbour (2001a; 2001b), for one, thinks that it tells us that time is illusory. It is argued that the fact that quantum states do not evolve under the superHamiltonian means that there is no change.

In reality the Einstein general relativity, results a mathematical time as the existence of an independent clock working in the physics in contrast with the reality of the time whereas we see here that the natural metric of the universe compatible with Mach's mechanics (Mach, 1960) should be fully governed by the mass distribution in the universe and then the speed should be fully governed by measurable universe (fully variable light speed universe) whereas we see that the Einstein general relativity is not completely on the measurable universe which was the dream of the Einstein to establish a fully Machian type of general relativity as followed by Brans and Dicke (1961) and some others.

Relevance of Planck scales with space time is still under debate and some scientists are in doubt about the combination of the quantum gravity and Planck units as questioned by Diego Meschini (2007) that:

"Is dimensional analysis such a trustworthy tool as to grant us definite information about unknown physics without needing to look into Nature's inner workings, or has our faith in it become exaggerated?"

Someone welcome to continue on Planck depended quantum gravity and someone not, as noted by $\mathrm{Ng}$ (2003, p. 1) that:
"But by extrapolating the well-known successes of quantum mechanics and general relativity in low energy, we believe one can still make predictions about certain phenomena involving Planck-scale physics and check for consistency."

Whereas that Baez (2000) made welcome critical observations against the hypothetical relevance of the Planck length in a theory of quantum gravity and as noted by Baez (P. 180) that:

\section{"a theory of quantum gravity might involve physical constants other than $c, G, \ldots$. .".}

But we should notice that natural quantization of space-time by Planckian quantization is not in conflict with quantum mechanical theories of quantum gravity so that we can see that the minimum length deduced by quantum mechanical gravity is compatible with Planck's units. 
In fact, Heisenberg uncertainty principle as the essence of quantum mechanics works well on the gravity too in the Planck's scale. Heisenberg uncertainty principle is not in contrast with Planck's scales suppose it is common for derivation of minimum distance so that the wave functions of the quantum mechanics for motion of the electrons in the atoms does not violate natural orbit of Bohr generated by classical electromagnetism.

However now scientists have not found a welldefined theory of everything to unify the gravity and quantum mechanics, but each type of quantum mechanical gravity theories in the future will be agreement again with natural classical quantization of space-time in Planckian format, for that both are common and unified with Heisenberg uncertainty principle.

Then natural results here are not in contrast to the quantum field theories which strongly would be on the vector bosons, fermions and the well-known vertex coupling between bosons and fermions. This is the same wave and point particle complementary. Both are working independently, but unified and agreement means the quantum mechanics and classical natural quantization in Planck's scale.

In Mach's mechanics, the space is just light equivalent and pure elements of the space is not valid and then if we are not exact on the determination of the space it is uncertainty on the determination of the light position and this is the same quantum mechanics formulized by Schrodinger which may be generalized into a dynamical space as noted by Diego Meschini (2007) that:

"Although this is a seemingly sensible expectation, it need not hold true either, for a theory of quantum gravity may also be understood in less conventional ways. For example, not as a quantum-mechanical theory of (general-relativistic) gravity but as a quantum mechanical theory of empty spacetime ...".

By the way we want to show here the significance of the Planck values in quantization of space time in quantum gravity and we have shown that how the natural metric is agreement with the Planck natural units in combination of the Machian universe and Riemannian physics.

\section{There is no Preferred Clock and Meter but the Light is Preferred Reference}

In fact, there is no meter and second as the physical realities in the fundamentals of the physics. These are originated by light measurement. To measure the time, we use from the light and about the length too is the same. Just for near hand distances we use from the rigid meter but in far and exact measurements we use from the light. However, we have the concept of the space in thought but in reality as Mach's mechanics (Mach, 1960) we know that the space is just the light traveling observations and when there is no light there is no space. Space is the light and conceptual space is out of physics.

In fact, the distance is the path, the light is traveling and there is no time and, the physics is timeless and on the Zeno's paradox, the motion should be certainly a quantum and displacement naturally should be an on-moment occurrence. The time is iteration and iteration is not fundamental but possible to assume a parameter as time $t$ that:

$d l=c d t$

This is showing an equation of three parameters which certainly in Mach's mechanics this is a tripartite equivalency of the parameters for that there is no any absolute scale for measuring the time and meter and the light speed, suppose all are relative together and dependent and these parameters are defining each other in a tripartite equivalency divided to the four realizable quantum modes that:

a. Light speed c considered as a universal invariant

b. Time dt considered as a universal invariant

c. Length dl considered as a universal invariant

d. Mathematical constraints to the time and place like the Einstein general relativity

When a parameter is being considered as a constant, then other parameters in the equation are closer to each other. In fact, we have tripartite equivalencies of the light speed and time and the light speed and length and also equivalency of the time and length, similar to the equivalency of the mass and energy, when the light speed is assumed as a constant in especial relativity.

\section{Natural Metric Tensor of the Universe}

The Tripartite equivalency of the parameters is caused by fully relativistic measurement of the parameters 1 and $t$ and $c$. Then in natural metric tensor of the universe we need just to know about the light speed and the light speed in each point of the universe will show the metric there and against the Einstein assumption about a conceptual clock, there is no any clock in the nature suppose when we know the light speed in a point of the universe we know the metric tensor there.

All the speeds are possible to define by a speed in relational dynamics and then just a speed can be considered for definition of the time as the light speed which it is preferred reference. This means that the time is a relative duration by the light to travel in the length scale and strongly there is no other way naturally.

This problem is describable by mathematics for that in the algebraic in an equation of several parameters, one 
of them can be considered as an independent parameter which it is used in parametrization of the equations and other parameters will be dependent to the assumed independent parameter as a very simple case of the Brouwer fixed-point theorem. Then the definition of the speed and time and meter independently on the metric tensor is not valid in general for nature and natural metric of the universe is a metric that it is equivalent with definitive equation of the motion $d l=c d t$ and a kind of this metric tensor is below metric tensor that:

$d s^{2}=c^{2} d t^{2}-d l^{2} \mid d l^{2}=d r^{2}+r^{2} d \theta^{2}$

This is the same Minkowski metric we can consider it in each point locally means differentially.

This metric tensor is general metric of the universe and the geodesy should be derived by a variable light speed form of the Minkowski metric and then naturally the universe metric tensor is the generalized Minkowski metric in variable light speed universe (VSL).

On the other hand, on the Mach's mechanics (Mach, 1960), the light speed too should be fully governed by total material existence of the universe and the metric fully governed by all matter of the universe was too the demand of the Einstein as noted by Einstein in a letter to de Sitter 1917 that:

"in my opinion it would be dissatisfying, if there were a conceivable world without matter. The $g^{-\mu v}$-field should rather be determined by the matter and not be able to exist without it. This is the heart of what I understand by the demand for the relativity of inertia. One could just as well speak of the 'material conditionedness of the geometry'. As long as this demand was not fulfilled, for me the goal of general relativity was not yet completely achieved. This was first achieved through the introduction of the $\lambda$ term."

As mentioned by Einstein himself it was realized that the Einstein general relativity is not achieved fully with Mach's inertia principle for that his total field concerns to the absolutist boundary conditions for being solved mathematically as noted in the Barbour book 'Mach's principle: From Newton's Bucket to Quantum Gravity' (1995) that:

"The goal of Einstein's (1917) famous cosmological paper was to eliminate the need to posit Minkowskian boundary conditions for the metric tensor in general relativity, for Einstein held that such boundary conditions violated the Machian requirement that the inertia of a body be fully determined by other masses alone."

In a rigorous criterion, the Laue (1921, P. 180) discussed the Einstein general relativity as noted that:

"according to the fundamental idea of the general theory of relativity, the inertia of a single body should vanish if it is at a sufficient distance from all other masses. for inertia can only be a relational concept, which can be applied only to two or more bodies. ... with the boundary conditions mentioned, however the inertia continues to exist. Such considerations have led Einstein to the hypothesis of a space which runs back on itself like the surface of a sphere."

By the way, on the tripartite equivalency of the parameters $l$ and $t$ and $\mathrm{c}$, satisfying the relation $d l=c d t$ it is resulted that the light speed is fully determining the metric tensor of the universe and then all components of the metric tensor of the universe should be the functions from the light speed and then we have a generalized natural isotropic metric tensor that:

$d s^{2}=F(c) d t^{2}-G(c) d l^{2} \mid c^{2}=\frac{F(c)}{G(c)}$

Functions $F$ and $G$ are arbitrary functions variable by light speed in general but we will see that these functions will be limited to some quantum functions in composition to the quantum units of the Planck and Mach's mechanics (Mach, 1960).

We should notice that when the light speed in the universe is fully determining metric tensor then we need no to use a hypothetical field violating the principia that:

- Light speed in each point of the universe determines fully the metric tensor

In reality if we consider a tensor field for gravity, then again in the answer we can transfer equivalently the time and light speed and meter because of natural tripartite equivalency of these parameters so that such a transformation has been used too in Schwarzschild (1916) as $R^{3}=r^{3}+\alpha^{3}$.

\section{Natural Derivation of Especial Theory of Relativity in the Mode of Light Speed Constancy}

In fact, there is no meter and second. In each inertial system, the light speed is constant means not variable for constancy of the gravitational potential energy. But 
problem is that in the inertial systems we have relative speed $\mathrm{v}$ between event and observer. Einstein did consider the principle of light speed constancy as an evident fact whereas the light speed constancy is argued by equivalency of the parameters time $t$ and light speed c. We can consider variable time and the length in a constant light speed universe argued in the Absolute Relativity (Lutephy, 2012a) as noted in this book that:

"In a simultaneous system, difference at light
speeds is equivalent with light speed
constancy at different times in non-
simultaneity."

Einstein did consider mistakenly a pure independent measurer as the clock and then he introduced the light speed constancy as an evident improvable fact in the nature whereas there is no any absolute clock independent of the light speed and there is no time unless the light and working of a mathematical clock independent of the light evidences is not natural even if it is correct mathematically. The reality is that for equivalency of the parameters we can assume the light speed as a constant and variation of the light speed in the relative observers will be transferred equivalently to the variation of the time and length parameters as it has been argued completely in the book Absolute Relativity (lutephy, 2012a).

\section{Light Speed Fully Governed by Gravitational Potential Energy}

\section{The Quantum Mode of Length Invariant Universe}

Light speed is constant just in a constant potential energy as noted by Einstein himself that:

"The principle of the constancy of the speed of light can be kept only when one restricts oneself to space-time regions of constant gravitational potential."

In variable light speed universe If we consider $\mathrm{dl}$ as a universal invariant then the general isotropic metric of the universe is:

$$
d s^{2}=c^{2} d t^{2}-d l^{2}
$$

This metric is showing a constant meter tensor aligned to a variable light speed and time form of the universe.

Now it needs to know about the light speed variation in the universe.

In reality minimum distance which the light is possible to jump is the Planck length (Planck, 1899) that:

$l_{p}^{2}=\frac{G}{c^{3}} \hbar$
Then because of quantization, $\mathrm{dl}$ in fundamental face should be replaced with the Planck length and then the mode of $l_{p}$ invariant universe is showing that:

$\chi_{l}=\frac{G}{c^{3}}=\frac{G_{0}}{c_{0}^{3}}$

Which $\chi_{l}$ is a universal constant.

And zero indexes $\left(G_{0}, c_{0}\right)$ are internal gravitational coefficient and light speed in the observer reference frame and $(G, c)$ are relevant values in the event reference frame. For example, observations in the earth for us are domestic and looking to a galaxy from earth position is an exotic observation and this is not specialized for earth suppose in each point of the universe we have relevant domestic observation and exotic observations.

In reality there is a direct relation between the Planck natural units and Mach's mechanics. Planck natural units are derived by dimensional calculus on the physics fundamental constants defining each other which is showing that Planckian quantum physics is a Machian universe. In Machian universe there is no conceptual elements and then Machian universe is a self-consistent universe that all parameters are defining each other cyclically and then each fundamental constant in the Machian universe is not absolute suppose is defined by other relevant constants which such a manner is used in the determination of Planck natural units and this reality was realized too by Dirac (1938) as noted by AlfonsoFaus (2009) that:

"at cosmological scales the dimensionless
numbers should be all of order one. This is
something that Dirac proposed in 1937..."

Then Machian universe and Planck quantum physics are unified and we have combined Planckian and Machian and Einstein-Riemannian physics as three in one.

According to the Mach inertia principle when the coefficients $G$ and $c$ are in the reference frame of the event then we have that:

$m=m_{0} \frac{G}{c^{2}} \gamma \mid \gamma=\sum_{i} \frac{m_{i}}{r_{i}}$

When the observer is on the event, the field is all gravity and then observed mass $m_{0}$ is gravitational mass. But for exotic observer (the observer scales is not the same at the event observer), the mass $m$ is inertial mass.

According to the Einstein weak equivalence principle as the equality of the inertial mass and gravitational mass based on the equivalence principle of relativity, for a domestic observer we have:

$\frac{G}{c^{2}} \gamma=1$ 
This is the famous Einstein-Dirac-Brans-DickeSciama-Randall-Whitrow relation which internally is aligned with Einstein weak equivalence principle.

But exotically when the observer is out of the event we have breakdown of the weak equivalence principle as:

$$
m=m_{0} \frac{G_{0}}{c_{0}^{2}} \gamma
$$

So that $G_{0}$ and $c_{0}$ are gravitational $G$ and light speed $c$ in the inertial frame of the exotic observer (not at the event observer) accompanied with static mass $m_{0}$ in its local constant potential energy.

This Equation (9) is agreement in general relativity too as one of the general relativistic relations $m=m_{0} \frac{G_{0}}{C_{0}^{2}} \int \frac{\rho}{r} d V$ and for Equation (9) we may refer too to the $\mathrm{Ph}$. D. thesis of the Brans (1961) and many papers and the books, on the subject of the Mach's inertia principle.

By multiplication of the Equation (9) to the light speed $\mathrm{c}$ and mixing with $\chi_{l}$ at Equation (6) it is deduced:

$$
m c=m_{0} c_{0} \frac{G}{c^{2}} \gamma
$$

Then mixing with Equation (8) we obtain:

$$
m c=m_{0} c_{0}
$$

And so, in $l_{p}$ invariant universe, the momentum $\mathrm{mc}$ is invariant fundamentally.

When the light speed is variable then by invariance of the Planck length in this quantum mode, for Planck quantum of time $t_{p}$ we can consider an Euclidian uniform quantum of time $\delta t$ so that:

$$
c_{0}^{2}(\delta t)^{2}=c^{2} t_{p}^{2}
$$

Embedding Planck time unit $t_{p}=\sqrt{\hbar G / c^{5}}$ in this relation we have:

$$
\begin{aligned}
& c_{0}^{2}(\delta t)^{2}=\frac{\hbar G}{c^{3}} \\
& c_{0}^{2}(\delta t)^{2}=\hbar \chi_{l}
\end{aligned}
$$

And then $\delta t$ is invariant and invariance of the Euclidean $\delta t$ means it is correct to write that:

$s_{p}^{2}=c^{2} t_{p}^{2}-l_{p}^{2}$

Also by relations (6) and (9) and (11) we have:

$$
c=\frac{1}{\chi_{l} \gamma}
$$

And this is light speed fully governed by matter of the universe and we can result the invariance of the Planck length by inverse way too, starting from the reality that on the light geodesy, the momentum of light is invariant.

On the other hand, as noted by Alfonso-Faus (2009):

$$
\begin{aligned}
& \text { "If we choose the ratio } G / c 3=\text { constant and } \\
& \text { the momentum mc = constant we make } \\
& \text { constants the factors in front of the action } \\
& \text { integrals for gravity [2]. The constancy of the } \\
& \text { factor mc preserves classical mechanics. This } \\
& \text { ensures to be able to derive the Einstein field } \\
& \text { equations applying the action principle." }
\end{aligned}
$$

This may generate a bridge between our results and the results by Alfonso-Faus.

\section{Quantum Modes of the Gravity}

By Equation (3), the general natural metric tensor of the universe compatible with Planck natural units is:

$d s^{2}=\left(\frac{c}{c_{0}}\right)^{3-n} c_{0}^{2} d t^{2}-\left(\frac{c_{0}}{c}\right)^{n-1} d l^{2}$

As we can see that in Planck scale format we have:

$s_{p}^{2}=\left(\frac{c}{c_{0}}\right)^{3-n} c_{0}^{2} t_{p}^{2}-\left(\frac{c_{0}}{c}\right)^{n-1} l_{p}^{2}$

Then Euclidean universal constants are:

$\left\{\begin{array}{l}\delta l=l_{p} \sqrt{\left(\frac{c_{o}}{c}\right)^{n-1}} \\ \delta t=t_{p} \sqrt{\left(\frac{c}{c_{0}}\right)^{3-n}}\end{array}\right.$

Then we have:

$$
\left\{\begin{array}{l}
\delta l=\sqrt{\frac{\hbar G}{c^{3}}\left(\frac{c_{o}}{c}\right)^{n-1}} \\
\delta t=\sqrt{\frac{\hbar G}{c^{5}}\left(\frac{c}{c_{0}}\right)^{3-n}}
\end{array}\right.
$$

Then we have universal constant $\chi_{n}$ in $n$-mode gravities:

$\chi_{n}=\frac{G}{c^{n+2}}$ 
Assuming Equation (9) in generalized n-mode gravity we have that:

$m=m_{0} \frac{k_{n} G_{0}}{c_{0}^{2}} \gamma \mid k_{n}=$ const

By Equation (21) implied in the Equation (22):

$m=m_{0} \frac{c_{0}^{n}}{c^{n}} \frac{k_{n} G}{c^{2}} \gamma$

And then we have:

$m c^{n}=m_{0} c_{0}^{n}$

Showing that in n-mode Planck's scale based gravity we have universal invariance $m c^{n}$.

Then we can generalize the definition of the energy to the below relation in quantum n-modes of the gravity:

$E_{n}=m c^{n}$

This generalized energy yields to a generalized quantum work law so that:

$d E=\vec{f} \cdot d \vec{r} \times v^{n-2}$

By Equations (25) and (26) and the equation $d p=f d t$ it is deduced:

$c^{n} d m=d(m v) v^{n-1}$

And calculating mathematically we obtain:

$m=\frac{m_{0}}{\sqrt[n]{1-\left(\frac{v}{c}\right)^{n}}}$

In reality from Equation (28) we have:

$(m c)^{n}=\left(m_{0} c\right)^{n}+(m v)^{n}$

This is generalization of the Dirac equation.

In reality Equation (29) is written as:

$(m)^{n}=\left(m_{0}\right)^{n}+\left(m \frac{v}{c}\right)^{n}$

And on the base of the conservation of momentum and energy (Lutephy, 2012b) it is deduced that:

$m^{n}=m_{0}^{n}+m_{p h}^{n} \mid m_{p h}=m \frac{v}{c}$
So that $m_{p h}$ is dynamic mass of the moving bodies and this is showing that the addition of the static mass and dynamic mass is just linear in $n=1$ mode but it is Pythagorean in $n=2$ mode and generally in Fermat shape in $n$-modes gravity.

In reality in each inertial system because of constancy of the light speed it is possible to multiple $\mathrm{c}$ to an equation and changing relevant equations and just in the variable light speed universe this difference is realizable and then PlanckEinstein energy-frequency famous relation $\delta E=\hbar v$ is generalized in quantum n-modes of the gravity as:

$\delta E=c^{n-2} \hbar v$

And probably the dimension of the radiation is relevant to the quantum $n$ so that $n=1$ yields a linear radiation (like the electrons in the atoms) and $n=2$ yields a surface radiation (like the electromagnetic radiation of a charged accelerating body) and $n=3$ yields spherical radiation.

For inertial system, the light speed is constant and then definition of the energy equations can be transferred equivalently with multiplication to the parameter $\mathrm{c}$ and then on the generalization of the work law we have generalized the potential energy in n-modes gravity as:

$\varphi_{n}=c^{n-2} \varphi$

On the other hand, by Equations (24) and (22) and (21) it is deduced that:

$c^{n}=\frac{1}{k_{n} \chi_{n} \gamma}$

And generally for quantum n-modes gravity we have a unified apparatus included to relevant metric tensor that:

$\left\{\begin{array}{l}l_{p}=\sqrt{\hbar \chi_{n} c^{n-1}} \\ c t_{p}=\sqrt{\hbar \chi_{n} c^{n-1}} \\ d s^{2}=\left(\frac{c}{c_{0}}\right)^{3-n} c_{0}^{2} d t^{2}-\left(\frac{c_{0}}{c}\right)^{n-1} d l^{2} \\ E=m c^{n} \\ d E=\vec{f} \cdot d \vec{r} \times v^{n-2} \\ m=\frac{m_{0}}{\sqrt[n]{1-(v / c)^{n}}} \\ \delta E=c^{n-2} \hbar v \\ c^{n}=\frac{1}{k_{n} \chi_{n} \gamma} \\ \frac{k_{n} G}{c^{2}} \gamma=1 \\ \varphi_{n}=c^{n-2} \varphi\end{array}\right.$ 


\section{Gravitational Geodesies in Quantum Modes}

The equation for geodesic lines is:

$\frac{d^{2} x^{\lambda}}{d q^{2}}+\Gamma_{\mu v}^{\lambda} \frac{d x^{\mu}}{d q} \frac{d x^{v}}{d q}=0$

where, $\Gamma$ represents Christoeffel symbol and variable $q$ parametrizes the particle's path through space-time. Christoffel symbol depends only on the metric tensor $g_{\mu v}$ and variable $q$ is a constant multiple of the proper time $\tau$ for time like orbits and then we just can use from the face that:

$$
d s=c_{0} d \tau
$$

We have a total vector acceleration as the addition of the centrifugal and radial components that:

$$
\vec{a}=\frac{d^{2} \vec{r}}{d t^{2}}=\frac{d^{2} r}{d t^{2}}-r\left(\frac{d \theta}{d t}\right)^{2}
$$

General resolution of the natural metric tensor derived by Equation (17) yields for a static test mass as:

$$
\frac{d^{2} \vec{r}}{d t^{2}}=\frac{3-n}{2 n} \frac{c^{2}}{c^{n}} \frac{d c^{n}}{d r}
$$

Substituting light speed from Equation (34) in the relation (39) yields:

$$
\frac{d^{2} \vec{r}}{d t^{2}}=\frac{3-n}{2 n} \frac{c^{2}}{\varphi} g_{N}
$$

The gravity for static mass in each point in generalized VSL universe is $g_{N}$ and then we obtain a generalized Machian relation in quantum n-modes of the gravity as:

$$
\frac{2 n}{3-n} \varphi=c^{2} \mid n \neq 3
$$

And then:

$$
k_{n}=\frac{2 n}{3-n} \mid n \neq 3
$$

And then light speed in quantum n-modes gravity is:

$$
c^{n}=\frac{1}{\frac{2 n}{3-n} \chi_{n} \gamma} \mid n \neq 3
$$

Then for $n=2$ we have:

$$
\left\{\begin{array}{l}
l_{p}=\sqrt{\hbar \chi_{2} c} \\
c t_{p}=\sqrt{\hbar \chi_{2} c} \\
c^{2}=\frac{1}{4 \chi_{2} \gamma} \\
d s^{2}=c_{0} c d t^{2}-\frac{c_{0}}{c} d l^{2} \\
E=m c^{2} \\
d E=\vec{f} \cdot d \vec{r} \\
m=\frac{m_{0}}{\sqrt{1-(v / c)^{2}}} \\
\delta E=\hbar v \\
\frac{4 G}{c^{2}} \gamma=1
\end{array}\right.
$$

And then for $n=2$, the metric tensor is written in the polar coordinate system as:

$d s^{2}=c_{0} c d t^{2}-\frac{c_{0}}{c} d r^{2}-\frac{c_{0}}{c} r^{0} d \theta^{2}$

It is visible that the Einstein coefficient $\kappa$ in general relativity is equal to $\chi_{2}$ so that:

$\kappa=\frac{G}{c^{4}}=\chi_{2}$

From Equation (44), the light speed c is written as:

$c=\frac{1}{\sqrt{4 \kappa \sum_{i} \frac{m_{i}}{r_{i}}+4 \kappa \frac{M}{r}}}$

It is correct to assume that:

$c_{0}=\frac{1}{\sqrt{4 \kappa \sum_{i} \frac{m_{i}}{r_{i}}}}$

And repeating calculations we deduce:

$c \sim c_{0}\left(1-\frac{2 G M}{c^{2} r}\right)$

And then in the frame $(r, t)$ we have:

$d s^{2}=c_{0}^{2}\left(1-\frac{2 G M}{c^{2} r}\right) d t^{2}-\frac{1}{\left(1-\frac{2 G M}{c^{2} r}\right)} d r^{2}$

This is Schwarzschild metric in agreement with invariance of $m c^{2}$ in the universe and Einstein 
constant $\kappa$ is invariant here too. Of course we need to notice that this metric tensor is not compatible when $2 G M / c^{2} \sim 1$ suppose in this case it should be used the exact solution which is not never showing a relativistic type black hole.

In reality out the potential energy too is invariant on the light geodesic line for that:

$$
k m c^{2}=m \varphi
$$

So that $k$ is a constant and then we have:

$$
c^{2}=k \varphi
$$

And this is the same Machian relation.

For the mode $n=3$ it is deduced that:

$$
d \tau^{2}=d t^{2}-\frac{1}{c^{2}}\left(d r^{2}+r^{2} d \theta^{2}\right)
$$

And resolution for static mass is showing that:

$$
\vec{a}=0
$$

But resolution of the Equation (17) for light instead static mass shows that the light orbit is almost Newtonian if:

$$
k_{3}=3
$$

And then for $n=3$ we have that:

$c_{3}=\frac{1}{3 \chi_{3} \gamma} \mid \chi_{3}=\frac{G}{c^{5}}$

For $n<3$, the light speed is negative and this is showing that however mathematically it is possible to generalize the gravity to $\mathrm{n}$ modes but physically it is limited to three modes $n=1$ and $n=2$ and $n=3$.

For $n=1$ we see the Planck length invariant universe:

$$
l_{p}=\sqrt{\hbar \chi_{1}}
$$

For $n=2$ we see that $l_{p} t_{p}$ is universal constant so that:

$$
l_{p} t_{p}=\frac{\hbar G}{c^{4}}=\hbar \chi_{2}
$$

And for $n=3$ it is deduced:

$$
t_{p}=\sqrt{\hbar \chi_{3}}
$$

And we see that the Planck time $t_{p}$ is a universal invariant in $n=3$ and amazingly we see that the energy is $m c^{3}$ in the Planck time invariant universe.

We need to notice that in the Planck time invariant universe too, if the masses to be assumed included to the many particles, then we can see that the geodesy of the particles additionally can show a Newtonian gravity on the static mass too similar to a proposal by Cook (1976) as noted in his paper that:

"the random motion of a particle confined to a small volume generates a Newtonian-type gravitational force and that the acceleration of a Dirac electron in a stationary state leads to a gravitational force of the correct magnitude."

\section{Planck-Mach-Einstein and Riemannian Unified New Cosmology}

Uuniverse Expansion on the Variable Speed of Light (VSL) and Cyclical Transformation of Ether to Baryonic Matter and Baryonic Matter to ether and Quantum Tunnelling from Horizon to the Background of the Universe

The flatness problem on the Friedmann equations (1922) is showing that the total density of the universe is fine-tuned to a critical density and then it requires increment of the total matter of the universe by creation of the matter via vacuum whereas this is violating the conservation law of the mass and energy. But here we propose the expansion of the universe aligned to the conservation law of mass and energy.

The point velocity of the light in the universe is depended to the potential energy and, isotropy and homogeneity of the universe implies that the point velocity of the light is almost constant. But we can assume an additional group velocity relevant to the additional mass of the universe reasoning the universe expansion in the way of space expansion.

Then speed of the space or group velocity of the universe is possible to be calculated by the light speed in quantum gravity for that the space in Mach's universe is physically the light and then by Equation (34) we obtain the group velocity as:

$$
v_{g}^{n}=\frac{1}{\chi_{n} \gamma}
$$

And then we have:

$v_{g}=\frac{c^{3}}{G M} r$ 
And then it is resulted that:

$\frac{c^{3}}{G M}=H \mid v_{g}=H r$

This is very closer to Hoyle-Carvalho formula (Kragh, 1999; Carvalho, 1996), obtained in a different way and deduced by dimensional calculus by Valev (2009).

For Hubble sphere by equation of the Horizon $c=H R$ and substituting this equation in the Equation (62) it is deduced that:

$$
H^{2}=\frac{4 \pi}{3} G \rho
$$

By homogeneity of the universe and constancy of the expansion rate $H$ it is resulted that:

$$
G=\text { const }
$$

By constancy of $G$ and constancy of the $\chi_{n}$, we have:

$c=$ const

The density of the ordinary matter is decreased by universe expansion and this is inconsistent with homogeneity of the universe unless permanent creation of the observable baryonic matter in the whole of the universe.

In reality creation of the visible baryonic matter and flux of the universe expansion $j_{\exp }$ yields to the equation of continuity that:

$\frac{d m}{d t}+\nabla \cdot j_{\exp }=\varepsilon$

Then homogeneity of the universe is showing that the expansion rate of the universe necessarily depends to the density of the observable baryonic matter of the universe for that in this form, changing density does change the expansion rate and this cyclical mechanism yields the system ever to an equilibrium point:

$\frac{d m}{d t}+\nabla \cdot j_{\exp }=0$

Assuming the background ether being independent of the observable baryonic matter, then creation of the observable baryonic matter by background ether will not result an equilibrium on the point that $\varepsilon=0$ and then the density of background ether too should be ever correlated to the density of the observable baryonic matter. This is showing a permenent annihilation of the observable baryonic matter to the ether and ether to the observable baryonic matter cyclically until the system to be ever stable on the equilibrium point.

Now we find the physical mean of the Yin-Yang as the transformation of the dark to bright and bright to dark permanently in the universe (Fig. 1).

This cosmology is closest to the ether theory proposed by Dirac (1951). Dirac (1951) proposed an ether of particles in an article in Nature. He stated: "With the new theory of electrodynamics we are rather forced to have an aether."

The ether was proposed initially as a realistic absolute frame of reference and here we see that quantum tunneling of the matter from horizon to the background of the universe is not compatible unless creation at the absolute zeros background. Then microwave background radiation (CMB) will be a basis for measurement of the speed of bodies verified observationally by Cahill (2003). As noted in this paper that:

"...Michelson interferometers show
absolute motion effects when operated in
dielectric mode, as indeed such experiments
had indicated and analysis of the
experimental data showed that the
measured speeds were all consistent with
the Cosmic Microwave Background (CMB)
dipole-fit speed of $369 \mathrm{~km} / \mathrm{s} . "$

But how the observable matter is created permanently and annihilated permanently?

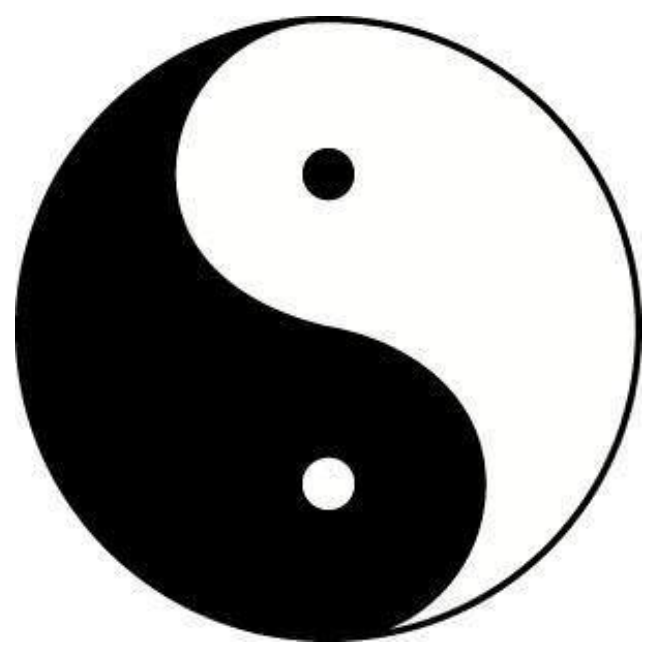

Fig. 1: The Yin-Yang symbol (showing cyclical correlation of the darkness and brightness) 
In reality it was deduced that the density and radius and mass of the universe all are invariant and then when the speed of the matter exceeds the point speed of the light $\mathrm{c}$ at the horizon, then it is unobservable because that commoving speed of the space is larger than the observable speed. But in Mach's mechanics there is no unobservable and then the rejected matter from the horizon for conservation of the mass and energy requires to return to the universe by a quantum tunneling from horizon to the space background and this is generating a cycle of the baryonic matter to ether and inverse.

Then creation of the matter is not from vacuum suppose the whole matter of the universe is invariant and what it is created it is annihilated at the horizon by Hubble law. In reality out of the radius of the universe, the matter returns to the universe by quantum tunneling creating an invariant universe in large scale accompanied with a stationary expanding universe (stop running universe) verifying isotropy and homogeneity of the universe and we name it a self-consistent universe.

Also by constancy of the $G / c^{3}$ and constancy of the momentum of universe $\mathrm{Mc}$ we see an argument for invariance of the universe in large scale by Alfonso-Faus (2009) "Artificial contradiction between cosmology and particle: The $\lambda$ problem" verifying our results here.

This mechanism is similar to mechanism of the life in the earth for human. Human is born in the earth and growing in the earth and dead ultimately but ever we have additionally a constant shape of the life (stop running) and in fact the life in the earth too is cyclical. Leave and return to the earth is necessity and we need to accept returning to the earth as we go and there should be a quantum tunneling for such a cyclical mechanism of the life. Then creation is not from vein but all are rotating in the universe and transforming together following conservation law of the existence similar to the existence of the comets which when they inter to the solar system, considered as the birth and when rejecting from the solar system, as the death in our observatories whereas that it is just ever living cyclical trajectory.

Even we have a similar mechanism in the biology so that we have ever creation and annihilation (speciation and extinction) like the annihilation of the dinosaurs and creation of some new species even visible newly. Also we can see that some species are being created suddenly and annihilated suddenly in thousands years which is short time in biology. Then in the biology too we have a quantum tunneling mechanism so that punctuated equilibrium (Eldredge and Gould, 1972) is a model of this rapid transition of the species.

Also creation of the matter in the universe background is a source ever for existence of the quantized minimum background temperature and this is the source of the microwave background radiation as a black body radiation.

In reality in $l_{p}$ invariant universe $(n=1)$ on the base of the Equation (62) we see that the constancy of the total matter of the universe implies the constancy of the Hubble constant in cosmic time.

Physicians are questioning the conclusion of accelerating expansion of the universe and they have a much larger dataset to back them up. When expansion rate is assumed to be constant along the billion years how it is changing in a decade scale?!

Now, a team of scientists led by Professor Subir Sarkar of Oxford University's Department of Physics has cast doubt on this standard cosmological concept. Making use of a vastly increased data set - a catalogue of 740 Type Ia supernovae, more than ten times the original sample size - the researchers have found that the evidence for acceleration may be flimsier than previously thought, with the data being consistent with a constant rate of expansion. The study is published in the Nature Journal Scientific Reports (Nielsen et al., 2016).

Of course we need to notice that the electric charge asymmetry is too affective on the variation of the expansion rate (Lutephy, 2019) because of antigravitational effect which may had a value on the universe expansion. then for permanent creation and annihilation of the matter in the universe, the Hubble expansion rate is fluctuating around a mean value by electric charge asymmetry in cosmic short scale like the decade, in agreement with observations for variation of the Hubble constant whereas if the universe was accelerating in a continuum shape in cosmic time then accelerating expansion was in contrast with long cosmic time of the constancy of the Hubble rate.

And in $n=2$ modes of the quantum gravity by Equation (61) we have:

$\frac{1}{c \chi_{2} M}=H \mid v_{g}=H r$

Then in this mode, the constancy of the total matter of the universe is not directly resulting the constancy of the expansion rate suppose light speed c too does change the expansion rate.

This is sowing a contraction of the universe if the expansion is accelerating because that by contraction of the universe, the light speed is decreased in agreement with the equation $c=H R$ and then expansion rate $H$ is increasing. But this is paradox and then the universe expansion is not accelerating unless by secondary effect like the anti-gravity. Then in $n=2$ mode of 
gravity too, the light speed should be constant for constancy of the expansion rate and also we see that the gravitational $G$ is constant for constancy of the light speed and then the density is too constant and universe is again expanding stationary.

\section{Newtonian Black hole and Absolute Equivalency of the Mass and Energy}

Newtonian black hole is real and Planck length is minimum distance in Newtonian type gravity too.

By a coincidence, the radius of a "Newtonian black hole" is the same as the radius of the Schwarzschild black hole in general relativity. We demand the escape velocity $\mathrm{v}$ to be the speed of light $\mathrm{c}$, so potential energy:

$\frac{G M m}{R}=\frac{m c^{2}}{2}$

But one must appreciate that these are totally different theories. In particular, there is nothing special about the speed $c$ in the Newtonian (nonrelativistic) gravity. To be specific, objects are always allowed to move faster than $\mathrm{c}$ which means that they may always escape the black hole and then in this manner, there are no real black holes in Newton's gravity.

But we want to argue here that the light speed is maximum too in Newtonian mechanics and the Newtonian black hole is real.

According to the work law we have:

$d E=f \cdot d r$

Simply on the vector calculus and the relation $d p=$ $f d t$ it is deduced that:

$d E=v d(m v)$

And integration results:

$$
\begin{aligned}
& \ln \left(m / m_{o}\right)=\ln \left|\left(c^{2}-v^{2} / c^{2}\right)\right|^{-\frac{1}{2}} \\
& m=\frac{m_{0}}{\sqrt{1-(v / c)^{2}}}
\end{aligned}
$$

Then in Newtonian gravity too we have a maximum speed $\mathrm{c}$ which the force can't produce it and again the energy and mass are equivalent without a necessity to the Einstein relativity and we have a Newtonian argument for Einstein formula $E=m c^{2}$.

Probably Einstein has been aware from this argument but it is clear that this argument destroys closer dependency of this famous relation to his relativity and in fact the Einstein several times tried to argue it in a relativistic way but all were failure. (Okun, 2008)

We see that the Newtonian type gravity will result black hole and black hole is not a general relativistic necessity.

\section{Explosion of the Light Around the Planck Density}

In loop quantum gravity theory, a Planck star is a hypothetical astronomical object that is created when the energy density of a collapsing star reaches the Planck energy density. Under these conditions, (in loop quantum gravity) assuming gravity and space time are quantized, there arises a repulsive force derived from Heisenberg's uncertainty principle. The accumulation of mass-energy inside the Planck star cannot collapse beyond this limit because it violates the uncertainty principle for space-time itself.

The existence of Planck stars was first proposed by Rovelli and Vidotto (2014), who theorized in 2014 that Planck stars form inside black holes as a solution to the black hole firewall and black hole information paradox.

The mass of the universe around the Planck density has a radius around the proton and this condition is compatible with the explosion of all the physical structures and explosion of the light. Then the density of the matter never could exceed from the Planck value suppose around the Planck density, the light is being exploded avoiding from collapse to singularity as Rovelli and Vidotto demonstrate that it may not have been emerging from a point of singularity as previously believed, but rather from the "bounce" of a Planck Star reaching the Planck density and therefore giving an alternative explanation to the so-called Big Bang.

Of course consideration of the quantum effects around the horizon is necessity like the entropy and uncertainty, opening doors for quantum mechanical gravity theories.

As (Sivaram, 1982), Preliminary matter of the universe initially had been all having the energy $E_{s}=$ $\hbar H$ coincides with the minimal measurable gravitational self-energy of a particle which is accepted as minimum quantum of energy from Alfonso-Faus (2012; Alfonso-Faus et al., 2013) and as (Gkigkitzis et al., 2013; Haranas and Gkigkitzis, 2013), this quantity takes substantial place in the estimations of total information and entropy of the universe and as a clue suggested by Weinberg (1972) which the large numbers are determined by both, microphysics and the influence of the whole universe, by division of the universe total mass to this candidate for smallest mass we deduce square of Dirac large number $\tilde{N}$ so that:

$\frac{M_{U}}{m_{s}}=\tilde{N}^{2}=\frac{c^{5}}{2 G \hbar H^{2}}$ 
Then not only the horizon is a quantum tunnelling for transformation of the baryonic matter to ether and inverse but in the inner of the universe too there is a next mechanism for such a cyclical transformation of the ether to the baryonic matter and inverse.

The ether is gradually transferring to the baryonic matter and inversely the baryonic matter too can regenerate the ether but not in a gradual way suppose when the baryonic matter reaches around the Planck density (Planck star), the baryonic matter can't resist the more and suddenly the photons are exploded transferring to the ether.

Then if the universe was not expanding, strongly it was ultimately collapsed to a super-giant black hole reaching to a density around the Planck density and then expanding universe avoids from explosion of the universe and this is one of the paradigms visible in the holy books too.

\section{Quantization of the Space-Time and Planckian Mini Universe}

As noted in the paper (Valev, 2014) Estimations of total mass and energy of the observable universe, a mass dimension quantity $m_{x}$ related to the universe could be constructed as:

$$
m_{x}=k c^{\alpha} G^{\beta} H^{\lambda}
$$

In reality on the dimensional calculus (Valev, 2013) it has been deduced three masses for universes as:

$$
\left\{\begin{array}{l}
m_{1}=\frac{c^{3}}{G H} \\
m_{2}=\frac{\hbar H}{c^{2}} \\
m_{3}=\sqrt[5]{\frac{H \hbar^{3}}{G^{2}}}
\end{array}\right.
$$

And manifestly the mass $m_{1}$ is the mass of our universe with radius $r_{1}$ so that $\mathrm{c}=H r_{1}$ is compatible with Mach inertia principle that:

$\frac{G}{c^{2}} \frac{m_{1}}{r_{1}}=1$

If in a sphere (volume V), the mass is somehow in agreement with Mach inertia principle that:

$\frac{G}{c^{2}} \frac{m_{i}}{r_{i}}=1$
This is showing that in that sphere, the potential energy prevails to the universal potential energy and then quantizing it and transferring it to a quasiuniverse which the internal equations are limited to the mass inside the boundary of the sphere and this is generating a quantum-universe which the potential energy is limited to the volume of the sphere and this universe is too expanding by its relevant Hubble constant $H$ that:

$c=H r_{i}$

Then on the dimensional calculus we have two next possibilities for fundamental universes appeared by the masses $m_{2}$ and $m_{3}$ in agreement with the Mach inertia principle.

Then for fundamental mass $m_{3}$ with radius of sphere $r_{3}$ if we have that:

$\frac{G}{c^{2}} \frac{m_{3}}{r_{3}}=1$

This is showing a quantum universe with radius $r_{3}$ which is expanding by relevant Hubble law and by this relation and the size of mass $m_{3}$ we deduce that:

$r_{3}=\frac{G}{c^{2}} \sqrt[5]{\frac{H \hbar^{3}}{G^{2}}}$

$r_{3}=l_{p}$

This is showing that the radius of this universe is Planck length and the mass $m_{3}$ is the same Planck mass for that by substituting the generalized Hubble law (Equation 79) into the mass $m_{3}$ we obtain that:

$m_{3}=\sqrt[5]{\frac{H \hbar^{3}}{G^{2}}}=\sqrt[5]{\frac{c}{l_{p}} \times \frac{\hbar^{3}}{G^{2}}}=\sqrt{\frac{\hbar c}{G}}=m_{p}$

Now for mass $m_{2}$, we have a quantum universe with radius $r_{2}$ so that:

$\frac{G}{c^{2}} \frac{m_{2}}{r_{2}}=1$

Then by the size of the mass $m_{3}$ we have:

$r_{2}=\frac{G}{c^{2}} \times \frac{\hbar H}{c^{2}}$

$r_{2}=l_{p}$ 
And by generalized Hubble law it is deduced that:

$m_{2}=\frac{\hbar H}{c^{2}}=\frac{\hbar c}{c^{2} r_{2}}=m_{p}$

This is showing that:

$m_{2}=m_{3}$

And then these two universes are just a universe with Planckian radius $l_{p}$ and Planckian mass $m_{p}$ similar to our universe with relevant expanding rate $H$ that:

$H=\frac{c}{l_{p}}=\sqrt{\frac{c}{\hbar G}}$

Then we need to accept the existence of the mini Planckian universes with radius $l_{p}$ so that because of the reality that it is minimum distance in our universe then the Planckian mini universe has no interaction with our universe unless by the effects of the entropy and uncertainty of quantum mechanics which allows to obvious horizontally and this coincidence of our universe with Planck scale universe has been suggested strongly in the results by Alfonso-Faus (2009) according to the correlation of the Planckian units to Dirac large numbers as noted in his paper "Quantization of the universe as a black hole" that:

"This strongly suggests that our universe can be considered to be an excited state of Planck's quantum black hole."

In reality on the quantum mechanical laws it is possible to obvious relevant signals from planckian mini universes and it seems the Galiver story is not a story in physics and we can observe some things come out from mini universes.

\section{Large Scale Obvious of Quantization}

Now consider a black hole as a quantum-universe. In this level because of quantization of universe into a black hole we have a large scale quantization of the space time.

For example, in $n=2$ mode of the gravity we have a variable Planck length as:

$l_{p}=\sqrt{\frac{\hbar G}{c^{3}}}=\sqrt{\chi_{2} \hbar c}$

And this is showing a variable Planck scale which it can use to obvious a large scale of the quantum by some technical ways on the information problem too.

\section{Conclusion}

The paper on the combination of the Planck and Mach and Einstein-Riemannian physics results natural metric of the universe fully governed by measurable matter of the universe and we have extracted quantum modes of the gravity included to the fundamental correlations between the fundamental constants and introducing new constants too. Also a new cosmology is being derived by the quantum results accompanied with the conservation of the mass and energy as a combination between quantum and cosmos.

\section{Acknowledgement}

The reader needs to refer to many papers published in the journals on the Planck units and its combination with large scale and referring to the Mach's mechanics is too necessity. We need to thanks from many physicists for their relevant publications which are out of number to quote.

\section{Ethics}

This article is original and contains unpublished material. The corresponding author confirms that all of the other authors have read and approved the manuscript and no ethical issues involved.

\section{References}

Alfonso-Faus, A., 2009. Artifical contradiction between cosmology and particle physics: The lambda problem. Astrophys. Space Sci., 321: 69-72.

DOI: 10.1007/s10509-009-0007-9

Alfonso-Faus, A., 2012. Universality of the selfgravitational potential energy of any fundamental particle. Astrophys. Space Sci., 337: 363-365.

DOI: $10.1007 / \mathrm{s} 10509-011-0803-\mathrm{x}$

Alfonso-Faus, A., M.J. Fullana and I. Alfonso, 2013. Cosmic Background Bose Condensation (CBBC). Astrophys. Space Sci., 347: 193-196.

DOI: $10.1007 / \mathrm{s} 10509-013-1500-8$

Baez, J.C., 2000. Higher-Dimensional Algebra and Planck-Scale Physics. In: Physics Meets Philosophy at the Planck Scale, Callender, C. and N. Huggett (Eds.), Cambridge University Press, Cambridge, pp: 177-195.

Barbour, J., 2001a. On general Covariance and Best Matching. In: Callender and Huggett, Callender, C. and N. Huggett (Eds.), Cambridge University Press, Cambridge, ISBN-10: 0521664454, pp: 199-212.

Barbour, J., 2001b. The End of Time: The Next Revolution in Physics. 1st Edn., Oxford University Press, Oxford, ISBN-10: 0195145925, pp: 374. 
Brans, C., 1961. Mach's principle and a varying gravitational constant. Ph.D. Thesis, Princeton University.

Brans, C. and R. Dicke, 1961. Mach's principle and a relativistic theory of gravitation. Phys. Rev., 124: 925-925. DOI: 10.1103/PhysRev.124.925

Bronstein, M., 1936. Quantentheorie schwacher Gravitationsfelder. Phys. Z. Sowjetunion, 9: 140-157. DOI: $10.1093 / \mathrm{mind} / \mathrm{XLV} .178 .157$

Cahill, T.R., 2003. Absolute motion and quantum gravity. Arxiv: physics/0209013

Carvalho, J.C., 1996. Derivation of the mass of the observable universe. Int. J. Theoretical Phys., 34: 2507-2509. DOI: 10.1007/BF00670782

Cook, R.J., 1976. is gravitation a result of Mach's inertia principle? Nuovo Sim., B35: 25-34. DOI: $10.1007 / \mathrm{BF} 02726280$

Dirac, P.A.M., 1938. The cosmological constants. Nature, 139, 323, 1937 and Proc. Roy. Soc. London, A 165: 199-208. DOI: 10.1038/139323a0

Dirac, P.A.M., 1951. Is there an aether? Nature, 168: 906-907. DOI: 10.1038/168906a0

Eddington, A., 1918. Report on the Relativity Theory of Gravitation. 1st Edn., Fleetway Press, London.

Einstein, A., 1917. Kosmologische Betrachtungen zur allgemeinen Relativitutstheorie. Sitz. Knig. Preuss. Akad. 142-152. Available in English translation as 'Cosmological considerations on the general theory of relativity' in (Lorentz et al.1952): 175-188. Also available in CPAE 6 (Doc. 43).

Eldredge, N. and S.J. Gould, 1972. Punctuated Equilibria: An Alternative to Phyletic Gradualism. In: Models in Paleobiology, Schopf, T.J.M. (Ed.), Freeman Cooper, San Francisco, pp: 82-115.

Friedmann, A., 1922. -ber die Krümmung des Raumes. Zeitschrift für Physik, 10: 377-386. DOI: $10.1007 / \mathrm{BF} 01332580$

Gkigkitzis, I., I. Haranas and S. Kirk, 2013. Number of information and its relation to the cosmological constant resulting from Landauer's principle. Astrophys. Space Sci., 348: 553-557.

DOI: $10.1007 / \mathrm{s} 10509-013-1581-4$

Haranas, I. and I. Gkigkitzis, 2013. Bekenstein bound of information number $\mathrm{N}$ and its relation to cosmological parameters in a universe with and without cosmological constant. Mod. Phys. Lett. A, 28: $1350077-1350079$.

DOI: $10.1142 / \mathrm{S} 0217732313500776$

Hawking, S.W., 1975. Particle creation by black holes. Commun. Math. Phys., 43: 199-220.

DOI: $10.1007 / \mathrm{BF} 02345020$

Kragh, H., 1999. Cosmology and Controversy: The Historical Development of Two Theories of the Universe. 1st Edn., Princeton University Press, Princeton, ISBN-10: 069100546X, pp: 212.
Laue, M., 1921. Die Relativitdtstheorie. Vol. 2: Die allgemeine Relativitdtstheorie und Einsteins Lehre von der Schwerkraft. Friedrich Vieweg und Sohn, Braunschweig.

Lévi, R., 1927. Théorie de l'action universelle et discontinue. J. Phys. Radium, 8: 182-198. DOI: 10.1051/jphysrad:0192700804018200

Lutephy, M., 2012a. Absolute Relativity: Equivalency of Time and Light Speed and Natural Relativistic Dynamics of Moving Bodies. 1st Edn., ISBN-13: 978-1091484795.

Lutephy, M., 2012b. Equivalency of momentum and kinetic energy and Pythagorean conservation of mass and energy. Int. J. Applied Phys. Math., 2: 296-300. DOI: 10.7763/IJAPM.2012.V2.117

Lutephy, M., 2019. Evidences of the charge fundamental asymmetry. CGG, 49: 67-107.

DOI: $10.2478 /$ congeo-2019-0005

Mach, E., 1960. The science of mechanics. Open Court.

Martin, J., 2001. Brandenberger RH. trans-Planckian problem of inflationary cosmology. Phys. Rev. D, 63: 123501-123501.

DOI: 10.1103/PhysRevD.63.123501

Mead, CA., 1964. Possible connection between gravitation and fundamental length. Phys. Rev., 135: 49-62. DOI: 10.1103/PhysRev.135.B849

Mead, CA., 1966. Observable consequences of fundamental-length hypotheses. Phys. Rev., 143: 990-1005. DOI: 10.1103/PhysRev.143.990

Meschini, D., 2007. Planck-scale physics: Facts and beliefs. Foundat. Sci., 12: 277-294.

DOI: $10.1007 / \mathrm{s} 10699-006-9102-3$

Ng, Y.J., 2003. Selected topics in Planck-scale physics. Modern Phys. Lett. A, 18: 1073-1097.

DOI: $10.1142 / \mathrm{S} 0217732303010934$

Nielsen, J.T., A. Guffanti and S. Sarkar, 2016. Marginal evidence for cosmic acceleration from Type Ia supernovae. Scientific Rep., 6: 35596-35596. DOI: $10.1038 /$ srep35596

Okun, L.B., 2008. The einstein formula: $E=m c 2$. Isn't the lord laughing? Physics-Uspekhi, 51: 513-527. DOI: 10.1070/PU2008v051n05ABEH006538

Planck, M., 1899. Naturlische Masseinheiten. 1st Edn., Der Koniglich Preussischen Akademie Der Wissenschaften, pp: 479.

Pokrowski, G.I., 1928. Zur Frage nach der Struktur der Zeit. Z. Phys., 51: 737-739.

DOI: $10.1007 / \mathrm{BF} 01331957$

Rovelli, C. and F. Vidotto, 2014. Planck stars. Int. J. Modern Phys. D, 23: 1442026-1442026. DOI: $10.1142 / \mathrm{S} 0218271814420267$

Rovelli, C., 2007. Quantum Gravity. In: Philosophy of Physics, J. Butterfield and J. Earman, (Eds.), Elsevier, Amsterdam, pp: 1287-1329. 
Schwarzschild, K., 1916. Uber das gravitationsfeld eines massenpunktes nach der einsteinschen theorie. Sitzungsberichte der Koniglich Preussischen Akademie der Wissenschaften, 7: 189-196.

Sivaram, C., 1982. Cosmological and quantum constraint on particle masses. Am. J. Phys., 50: 279-279. DOI: $10.1119 / 1.12870$

Unruh, W.G., 1995. Sonic analog of black holes and the effects of high frequencies on black hole evaporation. Phys. Rev. D, 51: 2827-2838. DOI: $10.1103 /$ PhysRevD.51.2827

Valev, D., 2009. Determination of toral mechanical energy of the universe within the framework of the Newtonian mechanics. Comput. Rend. Acad. Bulg. Sci.
Valev, D., 2013. Three fundamental masses derived by dimensional analysis. Am. J. Space Sci., 1: 145-149. DOI: 10.3844/ajssp.2013.145.149

Valev, D., 2014. Estimations of total mass and energy of the universe. Phys. Int., 5: 15-20.

DOI: $10.3844 /$ pisp.2014.15.20

Weinberg, S., 1972. Gravitation and Cosmology: Principles and Applications of the General Theory of Relativity. 1st Edn., Wiley, New York, ISBN-10: 0471925675, pp: 657. 